\title{
The influence of various small plasticisers and malto-oligosaccharides on the retrogradation of (partly) gelatinised starch
}

\author{
A.L.M. Smits ${ }^{\mathrm{a}, *}$, P.H. Kruiskamp ${ }^{\mathrm{a}}$, J.J.G. van Soest ${ }^{\mathrm{b}}$, J.F.G. Vliegenthart ${ }^{\mathrm{a}}$ \\ ${ }^{a}$ Bijvoet Center for Biomolecular Research, Utrecht University, P.O. Box 80075, NL-3508 TB Utrecht, The Netherlands \\ ${ }^{\mathrm{b}}$ Agrotechnological Research Institute ATO, P.O. Box 17, NL-6700 AA Wageningen, The Netherlands
}

Received 10 April 2002; accepted 15 July 2002

\begin{abstract}
Ageing of gelatinised and partly gelatinised potato starch and wheat starch were investigated in the presence of plasticisers with increasing size and number of $\mathrm{OH}$ groups (ethylene glycol, glycerol, threitol, xylitol, glucose, and for potato starch also maltose). The influences of these plasticisers and of granular remnants (ghosts) on recrystallisation were determined by using X-ray diffraction. Recrystallisation of potato starch samples in the presence of plasticisers resulted in crystallinity indices of $\sim 0.5$. The largest reduction in potato starch recrystallisation is found for threitol $(4 \mathrm{OH})$ and xylitol $(5 \mathrm{OH})$. In the plasticiser range examined, the crystallisation inducing effect of granular potato starch remnants is reduced better when the plasticiser contains more $\mathrm{OH}$ groups. Wheat starch recrystallises to a lesser extent than potato starch, resulting in crystallinity indices of $\sim 0.4$. The results for wheat starch do not show clear trends for the influences of plasticiser size and of ghosts. The difference in behaviour of the two starches is probably caused by wheat starch having shorter amylopectin chains. Resulting from these shorter amylopectin chains, the remaining structure in wheat starch ghosts may resemble A-type crystallinity, making it more difficult to form B-type crystals. Alternatively, the trends as found for potato starch may occur, but are less manifest for wheat starch, due to the lower total extent of recrystallisation. Solid state CP/MAS NMR spectra of the wheat starch samples containing ethylene glycol were obtained, in order to compare completely and partly gelatinised systems. The spectra were identical, confirming that the ghost structures do not influence wheat starch recrystallisation. Apparently, wheat starch ghosts do not act as nuclei for crystallisation.

Similarly, the influence of various malto-oligosaccharides in combination with granular remnants (ghosts) was investigated on wheat starch ageing. Gelatinised and partly gelatinised wheat starch were plasticised with maltose, maltotriose, maltotetraose, maltopentaose or maltohexaose. This resulted in crystallinity indices of $\sim 0.2$, with the largest reduction in recrystallisation for maltotriose and maltotetraose. No trend was found for the influence of ghosts. The presence of ghosts did not influence the ${ }^{13} \mathrm{C}$ solid state HP/DEC NMR spectra. Less recrystallisation took place than with the previously mentioned smaller plasticisers that resulted in crystallinity indices of $\sim 0.4$. The finding that maltose was able to reduce retrogradation better than glucose could be of practical importance.

(C) 2003 Elsevier Science Ltd. All rights reserved.
\end{abstract}

Keywords: Starch; Plasticisers; Malto-oligosaccharides; Ageing; Crystallinity; X-ray diffraction

\section{Introduction}

Starch based foods are processed at high water contents, leading to (partial) starch gelatinisation, and low water content products. Starch is transformed from a semicrystalline granular material into a system containing granular remnants, or to an amorphous paste with no structure at all. During gelatinisation, starch granules swell, while losing their crystallinity, until starch is completely dissolved. At some point, non-crystalline swollen granuleshaped structures remain, named ghosts (Atkin, Abeyse-

\footnotetext{
* Corresponding author.
}

kera, \& Robards, 1998). Sugars, lipids, flavours, salt and other additives are used to improve the product perception and sometimes to control the staling process. This reduction of the retrogradation process is mainly empirical and therefore often ineffective. Plasticisers such as water and sugars are needed to enable starch destructuring during processing. Plasticisers lower the glass transition temperature of starch, which may cause the material to be above $T_{\mathrm{g}}$ at room temperature. Unfortunately, in that state amylose and amylopectin retrogradation can occur, which reduces the products shelf-life. Some plasticisers reduce retrogradation as compared with equal amounts of other plasticisers, such as water. Therefore, the influence of 
plasticisers such as water and sugars on the recrystallisation of gelatinised starch is important for the processing and the shelf-life of starch-based foods (Chiotelli, Rolée, \& Le Meste, 2000; Gudmundsson, 1994; Katsuta, Nishimura, \& Miura, 1992a,b; Kohyama \& Nishinari, 1991; Krog, Oleson, Toernaes, \& Joensson, 1989; Levine \& Slade, 1991). Especially for new products, e.g. low-fat baked foods, insufficient insight is available into the retrogradation process. Like in foods, retrogradation influences the processing and final product properties in non-food starch based products. Starch based coatings, adhesives and plastics become brittle in the course of time because of starch retrogradation. Water and other plasticisers influence this process, glycerol for example is reducing it (van Soest, de Wit, Tournois, \& Vliegenthart, 1994).

Low molecular weight carbohydrates, such as monosaccharides and malto-oligosaccharides, are significant components in many food products. Knowledge of their influence on the glass transition of foods has helped food scientists to control storage stability and food quality (Levine \& Slade, 1991). In bakery processes, low molecular weight malto-oligosaccharides are produced by enzymatic hydrolysis of starch by $\alpha$-amylase. These sugars are known to reduce retrogradation, when comparing systems with equal total amounts of plasticiser, by interfering with starch recrystallisation (Katsuta et al., 1992a,b; Léon, Durán, \& Benedito de Barber, 1997).

Changes in the molecular organisation in low water content starch-plasticiser systems during ageing, and the inhibiting effect of polyol plasticisers on the ageing process were investigated. Much research has been done on starch gels, but for relevance to bakery products low water content gelatinised starch systems are examined in the present study. The influence of granular remnants on retrogradation is interesting, since starch is often partly gelatinised when processing food products (Atkin et al., 1998; Levine \& Slade, 1991). Therefore, potato starch and wheat starch were fully or partly gelatinised in order to examine the influence of granular remnants on ageing. Small plasticisers were used with increasing size and number of $\mathrm{OH}$ groups (ethylene glycol, glycerol, threitol, xylitol, glucose, and for potato starch also maltose). Since it would be interesting to know if there is an optimum in the reduction in retrogradation with a certain malto-oligosaccharide chain length, the influence of maltose, maltotriose, maltotetraose, maltopentaose and maltohexaose was investigated on wheat starch ageing.

\section{Experimental}

\subsection{Sample preparation}

Gelatinisation is performed in a Brabender viscometer. A $41.86 \mathrm{~g}$ native potato starch, PN (Avebe), or $40.49 \mathrm{~g}$ wheat starch, WN (Amidon Haussy, Avebe), were mixed with de- ionised water to gain $350 \mathrm{~g}$ of a $10 \%$ dry weight starch dispersion. The $350 \mathrm{~g}$ mixture was poured into the Brabender and stirred at a frequency of $75 \mathrm{rpm}$, while heating from room temperature to $90{ }^{\circ} \mathrm{C}$ with $2{ }^{\circ} \mathrm{C} / \mathrm{min}$. The completely gelatinised mixture was obtained by keeping it at $90{ }^{\circ} \mathrm{C}$ for $55 \mathrm{~min}$, yielding a fully pasted sample. The partly gelatinised sample was prepared by stopping at the peak viscosity, which was reached for $\mathrm{PN}$ at $65^{\circ} \mathrm{C}$ and for WN after keeping it for $4 \mathrm{~min}$ at $90{ }^{\circ} \mathrm{C}$. The partly gelatinised samples were inspected with polarised light microscopy for the presence of ghost structures, and for the absence of granules. Granules would appear as typical Maltese crosses. Granular remnants appeared as noncrystalline ghost structures, as was examined with X-ray diffraction.

The gelatinised mixtures were dried under reduced pressure in a vacuum-oven for $24 \mathrm{~h}$ at $50{ }^{\circ} \mathrm{C}$. Plasticisers were added to the $\mathrm{PN}$ and $\mathrm{WN}$ samples in a ratio dry starch/plasticiser of 100:30 w/w. Solid plasticisers were dissolved in a small amount of water and mixed with the gel after drying for $\sim 3 \mathrm{~h}$, when it was still humid. Liquid plasticisers were mixed in after drying, in order to prevent evaporation during drying. In the first plasticiser range, plasticisers used were dry glycerol (Fluka), dry ethylene glycol (Acros), threitol and xylitol (Aldrich), glucose (Sigma), and maltose (Merck). Equal plasticiser weight ratios imply similar total numbers of hydroxy groups in the plasticiser and thus similar $\mathrm{OH}$ densities. In the second plasticiser range, plasticisers used were maltose (Merck), maltotriose, maltotetraose, maltopentaose and maltohexaose (Sigma). Equal plasticiser weight ratios were used in order to have added equal numbers of glucose residues, resulting in similar $\mathrm{OH}$ densities. The samples were ground after freezing with liquid nitrogen and conditioned for several weeks at $20{ }^{\circ} \mathrm{C}$ and $90 \% \mathrm{RH}$. Their crystallinity was measured using X-ray diffraction.

Results for erythritol (Aldrich) are not considered, because that plasticiser did not dissolve completely in the starch gel. In X-ray powder diffraction (XRD) intense sharp peaks were found characteristic of crystalline sugars (linewidth $<0.5^{\circ}(2 \theta)$ ), implying a phase-separated character. Only the dissolved part of erythritol acts as a plasticiser. The other plasticisers were homogeneously mixed with starch.

\subsection{Analyses}

Wide angle XRD was performed on a Philips PC-APD diffractometer in the reflection geometry in the angular range $4-40^{\circ}(2 \theta)$. The $\mathrm{CuK}$ radiation from the anode operating at $40 \mathrm{kV}$ and $50 \mathrm{~mA}$ was monochromised using a $15 \mu \mathrm{m} \mathrm{Ni}$ foil, filtering off the $\mathrm{Cu} \mathrm{K}_{\beta}$ radiation $(1.39 \AA)$ and letting through $\mathrm{Cu} \mathrm{K}_{\alpha}$ radiation $(1.54 \AA$ ). The diffractometer parameters were: divergence slit $1^{\circ}$, receiving slit $0.2 \mathrm{~mm}$ and scatter slit $1^{\circ}$. A proportional detector was used to detect the scattered radiation. Samples were initially 


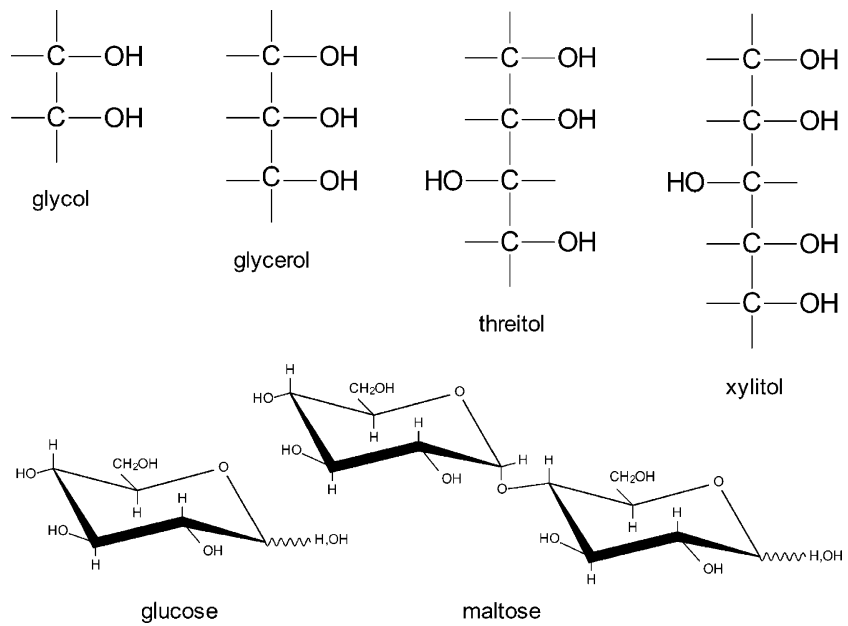

Fig. 1. Plasticisers varying in size and in the number of $\mathrm{OH}$ groups.

amorphous and retrogradation merely resulted in B-type crystallinity. Diffractograms were baseline corrected by drawing a straight line between the intensities at 7 and $40^{\circ}(2 \theta)$. The ratio of the height of the crystalline diffraction at $17.3^{\circ}(2 \theta)$ and the height of the total diffraction at this angle was defined as the crystallinity index, a measure for the B-type crystallinity of the sample.

Solid state cross-polarisation magic angle spinning (CP/MAS) and high power decoupling (HP/DEC) NMR spectra were collected on a Bruker AMX 400 spectrometer operating at $100.63 \mathrm{MHz}$ for ${ }^{13} \mathrm{C}$. Samples were spun at the magic angle $\left(54.7^{\circ}\right)$ with respect to the static magnetic field. Carbon chemical shifts relative to tetramethylsilane (TMS) were determined from the spectra, using solid glycine at room temperature as external reference. Samples were packed into $7 \mathrm{~mm}$ ceramic rotors and spun at $4 \mathrm{kHz}$. The recycle delay was set to $4 \mathrm{~s}$ and in CP/MAS experiments, the cross polarisation time was set to $500 \mu \mathrm{s}$.

\section{Results and discussion}

Retrogradation was examined on fully and partly gelatinised potato and wheat starch, using a range of small plasticisers with increasing size and number of $\mathrm{OH}$ groups (Fig. 1), and for wheat starch also by using a range of maltooligosaccharides with increasing number of glucose residues. The samples have a dry starch/plasticiser weight ratio of 100:30. Conditioning at $90 \% \mathrm{RH}$ and $20^{\circ} \mathrm{C}$ allows amylose and amylopectin retrogradation, the former occurring faster than the latter (van Soest, Tournois, de Wit, \& Vliegenthart, 1995). Within two days of conditioning, the samples are at equilibrium moisture content. By that time most of the recrystallisation as measured by X-ray diffraction has already taken place. Therefore, focus is on the final recrystallisation. Experiments are well reproducible with a spread of \pm 0.01 in crystallinity index.

Conditioning of the samples at $90 \% \mathrm{RH}$ and $20{ }^{\circ} \mathrm{C}$ results in equilibrium water contents of $\sim 30 \%$ (Bizot, Buléon, Mouhous-Riou, \& Multon, 1985). There may be small variations in the water contents in relation to the hygroscopicity of the plasticiser used (Lourdin, Coignard, Bizot, \& Colonna, 1997; Nirkko, Gál, Giovanoli, \& Signer, 1975). However, at these high water contents this will not have a large influence on retrogradation and will not outweigh the effect of the plasticiser used.

Less recrystallisation takes place when no plasticiser is added. This is due to the lower amount of plasticiser (including water). By consequence, the samples have a different condition with respect to their glass transition temperature. The samples with no plasticiser other than water have a glass transition temperature of $T_{\mathrm{g}}=10^{\circ} \mathrm{C}$. Whereas the samples that contain an additional plasticiser have a much lower $T_{\mathrm{g}} \sim-10{ }^{\circ} \mathrm{C}$ (Bizot et al., 1997; Kalichevsky, Jaroszkiewicz, \& Blanshard, 1993; Lourdin et al., 1997). Therefore, it is only possible to compare the influence of plasticisers other than water.

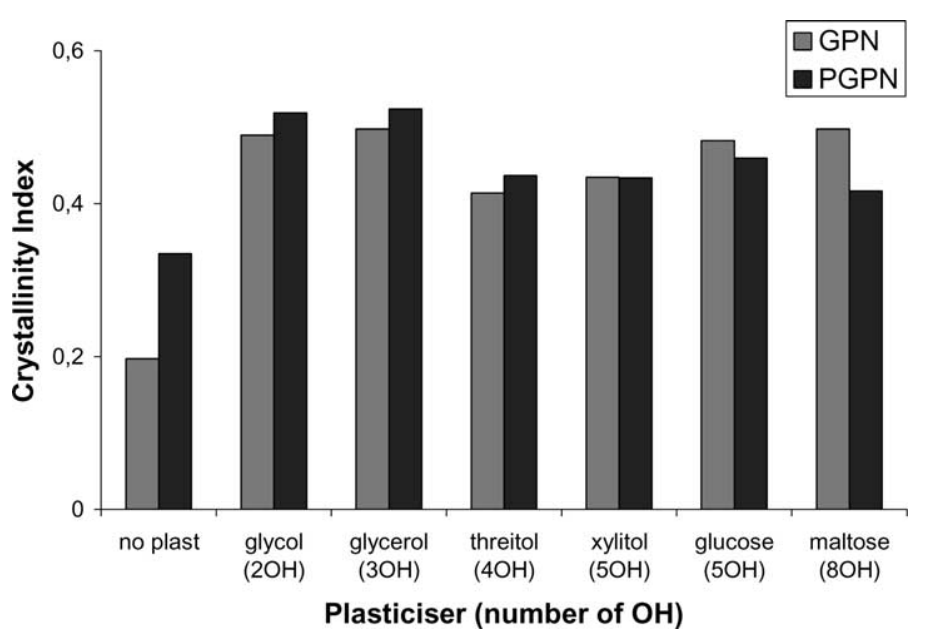

Fig. 2. Final recrystallisation of completely (GPN) and partly gelatinised (PGPN) potato starch without added plasticiser, and with plasticisers varying in size and in number of $\mathrm{OH}$ groups. 


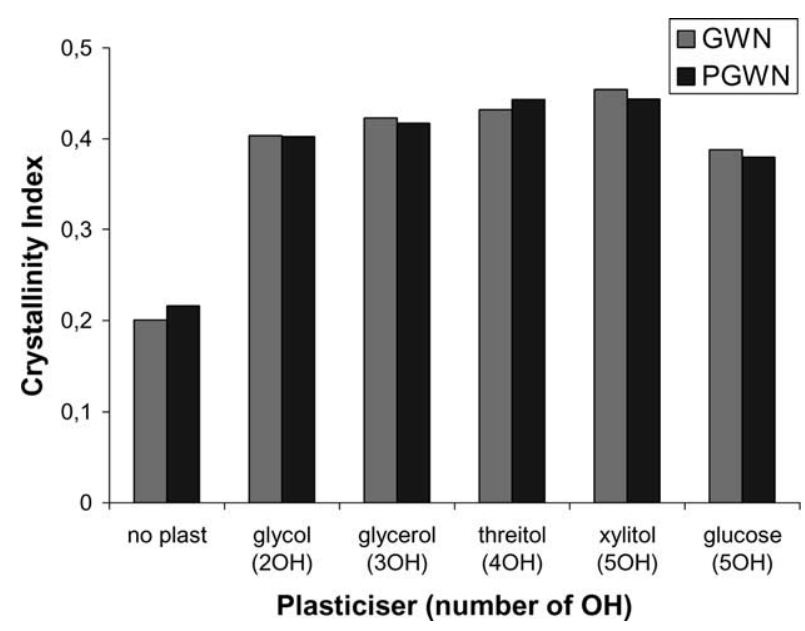

Fig. 3. Final recrystallisation of completely (GWN) and partly gelatinised (PGWN) wheat starch without added plasticiser, and with plasticisers varying in size and in number of $\mathrm{OH}$ groups.

\subsection{Small plasticisers}

\subsubsection{Potato starch recrystallisation}

As shown in Fig. 2, the recrystallisation of (partly) gelatinised potato starch reaches a crystallinity index of $\sim 0.5$. With increasing plasticiser size, the largest reduction in recrystallisation is found for threitol $(4 \mathrm{OH})$ and xylitol $(5$ $\mathrm{OH})$. For bakery or other food industry, the use of xylitol

(a)

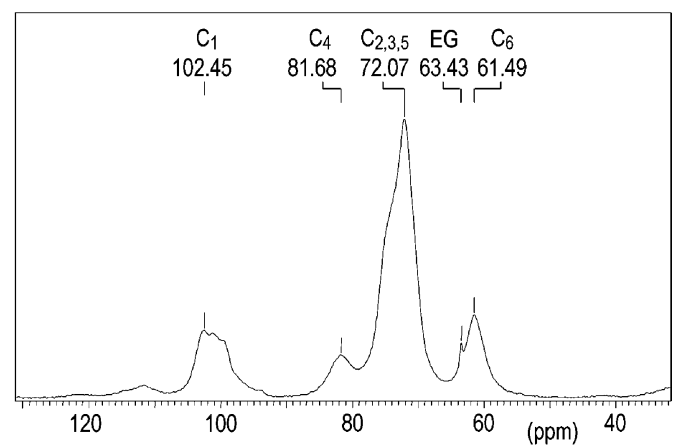

(b)

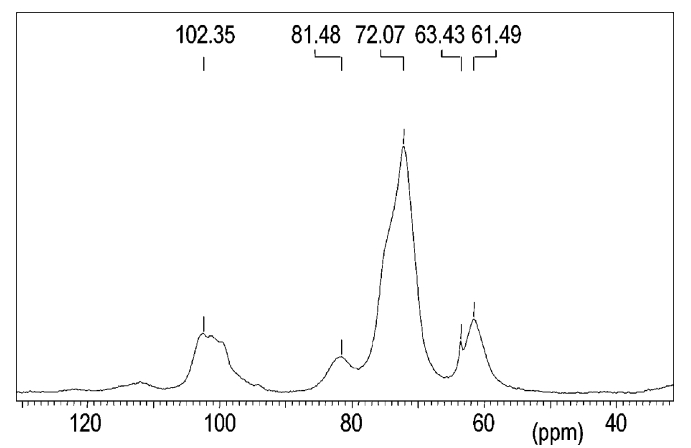

Fig. 4. Solid state ${ }^{13} \mathrm{C} \mathrm{CP} / \mathrm{MAS}$ NMR spectra of recrystallised partly (a) and fully (b) gelatinised wheat starch plasticised by ethylene glycol (63.4 ppm). instead of glucose would probably be too expensive for a small improvement in reducing retrogradation. In non-food products based on tuber starches (with B-type crystallinity), using threitol instead of glycerol can be interesting for reducing retrogradation. For controlled release applications of starch, the plasticiser trend is interesting because of the influence of crystallinity on the release behaviour. Crystallinity slows down release and is needed for capsule stability, but too high crystallinity causes capsules to crack when swelling or induces micropores in the capsule, increasing release (Ispas-Szabo, Ravenelle, Hassan, Preda, \& Mateescu, 2000; Miyajima, Koshika, Okada, Ikeda, \& Nishimura, 1997; Yadav, Khilar, \& Suresh, 1997).

Fully and partly gelatinised starch systems were compared, to investigate to which extent ghosts can act as nuclei for crystallisation. These ghosts are not crystalline, but amylopectin entanglements are holding the structure. These amylopectin structures may act as nuclei for crystallisation. When no plasticiser is added and for small plasticisers up to $4 \mathrm{OH}$ groups, crystallisation proceeds further for partly than for fully gelatinised potato starch samples, corresponding with crystal nucleation by ghosts. For larger plasticisers, however, more crystallisation takes place for fully than for partly gelatinised samples. The larger the number of $\mathrm{OH}$ groups in the plasticiser, the better the plasticiser reduces the crystallisation inducing effect of remaining ghosts. It may be, that the larger plasticisers that contain more $\mathrm{OH}$ groups interact with the ghost structures and obstruct interaction of the polysaccharide chains, thus preventing recrystallisation of the remaining ghost structure. The smaller, linear plasticisers are better capable of mobilising starch chains. They are therefore also capable of mobilising the remaining ghost structure, enabling crystal propagation at the ghost structures. It is clear that in both food and non-food applications of starch, granular remnants can influence starch retrogradation, the effect depending on the plasticiser used.

\subsubsection{Wheat starch recrystallisation}

Wheat starch recrystallises to a lesser extent than potato starch, resulting in crystallinity indices of $\sim 0.4$ (Fig. 3). In contrast to potato starch, there are no clear trends in the recrystallisation with increasing plasticiser size or with the presence of ghosts for (partly) gelatinised wheat starch. Amidon Haussy wheat starch is, like potato starch, pure and contains little fat and protein. However, the amylopectin structure is different from that of potato starch. Native potato starch has B-type crystallinity, whereas native wheat starch has A-type crystals. Amylopectin chains are shorter for A-type starches, whereas B-type starches have a longer distance between the branch points (Gérard, Planchot, Colonna, \& Bertoft, 2000; Hizukuri, 1985). In general, cereal amylopectin retrogrades to a lesser extent than pea and potato amylopectin, because of the shorter average chains of the cereal amylopectin (Fredriksson, Silverio, Andersson, Eliasson, \& Åman, 1998; Gudmundsson, 1994; 


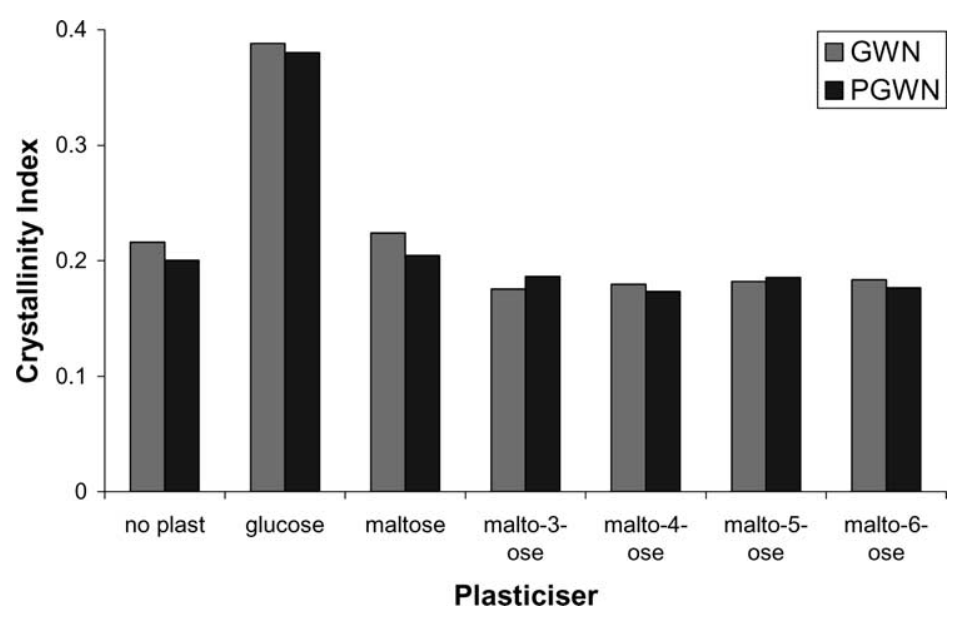

Fig. 5. Final recrystallisation of completely (GWN) and partly gelatinised (PGWN) wheat starch without added plasticiser, with glucose, and with a range of malto-oligosaccharides.

Kalichevsky, Orford, \& Ring, 1990; Silverio, Fredriksson, Andersson, Eliasson, \& Åman, 2000). Resulting from these shorter amylopectin chains, the remaining structures in wheat starch ghosts may resemble A-type crystallinity, which make it more difficult to form B-type crystals (Imberty, Buléon, Tran, \& Pérez, 1991; Waigh, Donald, Heidelbach, Riekel, \& Gidley, 1999). Since B-type crystals are formed during recrystallisation, the ghost structures can then not facilitate crystallisation, and no trend can be found with increasing plasticiser size. Alternatively, it may be that the trends as found for potato starch with increasing plasticiser size also occur for wheat starch, but are less manifest due to the lower degree of recrystallisation.

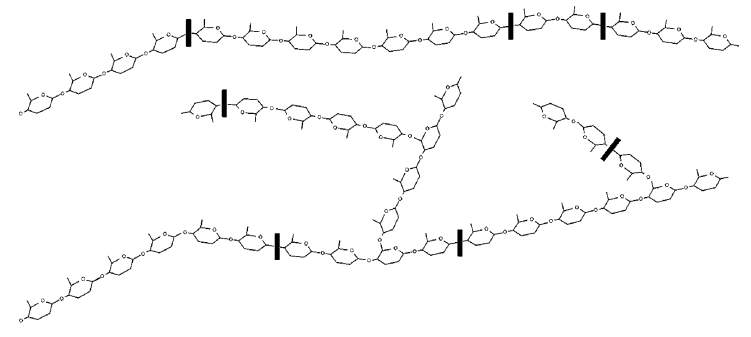

(a)

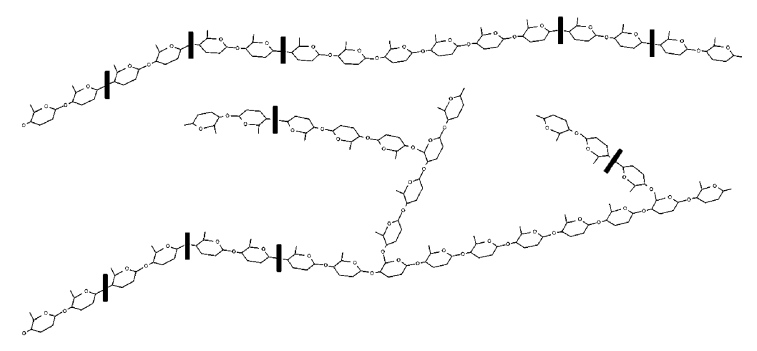

(b)

Fig. 6. Enzymatic action on starch, shown by I. 'Random' attack by $\alpha$ amylase (a) and external removal of maltose units by $\beta$-amylase (b).

\subsubsection{Solid state NMR spectroscopy}

Further studies were performed by solid state CP/MAS NMR spectroscopy on the aged wheat starch samples containing ethylene glycol to gain better insight into the recrystallisation process and to compare completely and partly gelatinised systems. An ethylene glycol signal appeared at $63.4 \mathrm{ppm}$ in the spectra of both preparations (Fig. 4). This implies that the plasticiser is partly immobilised because of its interaction with starch.

Identical spectra were obtained with identical relative ethylene glycol signals, implying similar molecular organisation independent of the presence of ghosts. There is no influence of wheat starch ghosts on the mobility of the plasticiser. Since there is no obvious influence of ghosts on the molecular mobility in the sample, presumably there will be no influence of ghosts on wheat starch recrystallisation, which confirms the recrystallisation results. Apparently, wheat starch ghosts do not act as nuclei for crystallisation. HP/DEC NMR spectra were obtained as well, but did not lead to additional information, because of the large amount of mobile ethylene glycol.

\subsection{Malto-oligosaccharides}

\subsubsection{Wheat starch recrystallisation}

Recrystallisation of wheat starch plasticised with maltose, maltotriose, maltotetraose, maltopentaose or maltohexaose is presented in Fig. 5. With this range of maltooligosaccharides, less recrystallisation takes place than with the smaller plasticisers ethylene glycol, glycerol, threitol, xylitol and glucose. They resulted in crystallinity indices of $\sim 0.4$, whereas the malto-oligosaccharide plasticised systems gave crystallinity indices of $\sim 0.2$. The maltooligosaccharides substantially reduce retrogradation. For comparison, the results for glucose have been added to Fig. 5. The largest reduction in recrystallisation of (partly) gelatinised wheat starch is found for maltotriose and maltotetraose. The larger malto-oligosaccharides reduce 


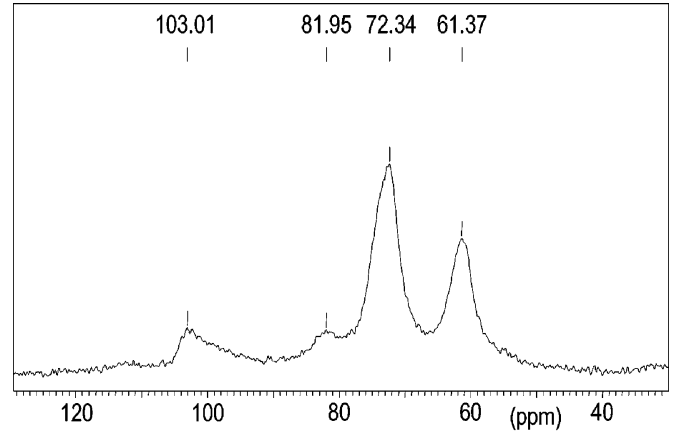

(a)

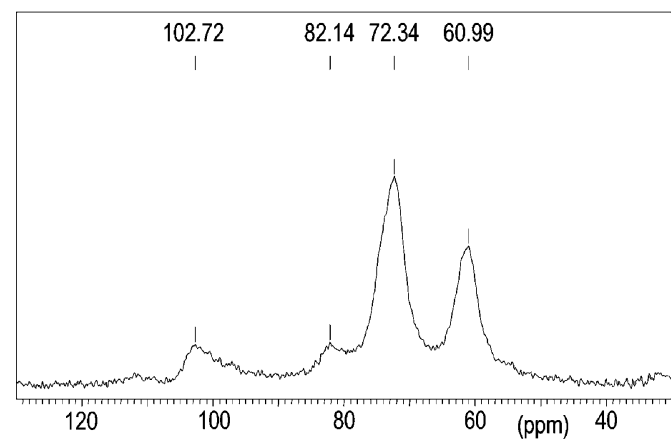

(b)

Fig. 7. Solid state HP/DEC NMR spectra of partly (a) and completely (b) gelatinised wheat starch containing maltohexaose.

retrogradation better than maltose. There is no obvious trend in the influence of ghosts on recrystallisation. A possible influence of ghosts may be not manifest, due to the low degree of recrystallisation.

Malto-oligosaccharides with a degree of polymerisation DP 3-6 reduce retrogradation better than maltose. However, these oligosaccharides are less available and more expensive than maltose. The limited reduction in retrogradation would therefore probably not justify the bulk application of these larger oligosaccharides. More importantly, maltose is found to reduce wheat starch retrogradation substantially better than glucose. This makes it useful to add maltose instead of glucose to bakery products, for the prevention of retrogradation. It would be advisable to use an amylase type that cuts back up to maltose, but that does not create glucose, for example a $\beta$-amylase (Nilsson et al., 2001). Whereas $\alpha$-amylase 'randomly' hydrolyses internal $(1 \rightarrow 4)$ bonds, finally producing glucose, maltose and small branched oligosaccharides, $\beta$-amylase removes maltose units in a stepwise fashion at external $(1 \rightarrow 4)$ linkages, without hydrolysing or bypassing branch points (Fig. 6).

\subsubsection{Solid state NMR spectroscopy}

Solid state HP/DEC NMR spectra were obtained from partly and completely gelatinised wheat starch with maltopentaose and maltohexaose to gain better insight into the recrystallisation process. No clear differences were found between completely and partly gelatinised systems (Fig. 7), implying that there is no influence of wheat starch ghosts on the mobility of the malto-oligosaccharide. Since ghosts do not seem to influence the molecular mobility in the sample, presumably there will be no influence of ghosts on wheat starch recrystallisation, which confirms the recrystallisation results. CP/MAS NMR spectra did not lead to additional information, because the malto-oligosaccharides could not be revealed, due to complete overlap with the starch signals.

\subsubsection{Influence of chain length}

Recently, Durán et al. reported on the influence of maltooligosaccharides on gelatinisation and retrogradation as studied by DSC (Durán, Léon, Barber, \& Benedito de Barber, 2001). Their systems probably recrystallised more than the systems reported here, because of the higher amount of water (1 starch:2 water). Amounts of oligosaccharide were used ( $27 \%$ on starch base) that are comparable with the amounts in the present study. In wheat starch solutions with malto-oligosaccharides with DP 2-7, they found that up to DP5 the extent of retrogradation is reduced. At DP6 more retrogradation occurred than with DP5 but retrogradation was still reduced. And with DP7 retrogradation increases. Their results show a minimum in retrogradation at maltopentaose.

Starch crystal helices contain six sugar rings per helix turn. Sugiyama et al. showed that malto-oligosaccharides up to DP5 have conformation different from longer maltooligomers, giving slightly different angles between the contiguous glucose residues (Sugiyama et al., 2000). This may be related to the reduction of recrystallisation of starch plasticised by malto-oligosaccharides up to DP6, and the increase in recrystallisation from DP7 onwards. The small malto-oligosaccharides interact with the starch chain, and may thus hinder helix formation. The larger the maltooligosaccharide (DP 2-5), the better it reduces recrystallisation. Malto-oligosaccharides of DP6 can form just one helix turn, and as such may be less able to reduce retrogradation. The larger oligosaccharides (DP $>6$ ) may form small helices that can be incorporated into the starch helix and increase retrogradation. This is confirmed by Gidley et al. who reported that for pure malto-oligosaccharides, the minimum chain-length required for crystallisation is DP10, but in the presence of longer chains, maltooligomers as short as maltohexaose can co-crystallise (Gidley \& Bulpin, 1987).

\section{Conclusions}

Potato starch and wheat starch were fully or partly gelatinised, and aged in the presence of a range of small plasticisers. The largest reduction in potato starch recrystallisation is found for threitol $(4 \mathrm{OH})$ and xylitol $(5 \mathrm{OH})$. 
The larger the number of $\mathrm{OH}$ groups in the plasticiser, when going from ethylene glycol to maltose, the better the plasticiser reduces the crystallisation inducing effect of remaining structure (ghosts) in potato starch. Up to $4 \mathrm{OH}$ groups (the turning point), more recrystallisation takes place when ghosts are present. Smaller plasticisers facilitate crystallisation nucleated by ghosts, by mobilising the starch chains. Larger plasticisers intrude between the starch chains and thereby prevent crystal propagation. It is important for both food and non-food applications of starch that granular remnants can influence retrogradation, however, the effect is dependent on the plasticiser used.

Wheat starch recrystallises to a lesser extent than potato starch, probably because of the shorter amylopectin chains (Fredriksson et al., 1998; Kalichevsky et al., 1990; Silverio et al., 2000). Recrystallisation of wheat starch does not show clear trends with increasing plasticiser size and number of $\mathrm{OH}$ groups in the range of ethylene glycol tot glucose. Solid state CP/MAS NMR spectra of wheat starch samples containing ethylene glycol confirm that the ghost structures do not influence wheat starch recrystallisation. Especially in non-food products, e.g. controlled release systems, based on tuber starches (with B-type crystallinity), the use of threitol instead of glycerol can be interesting for reducing retrogradation.

Malto-oligosaccharides substantially reduce wheat starch recrystallisation. No trend was found for the influence of remaining granular structure (ghosts). The trend with increasing malto-oligosaccharide chain length relates to the number of glucose residues needed for helix formation. Malto-oligosaccharides of DP 2-5 may hinder helix formation, and are thus able to reduce retrogradation. Whereas larger malto-oligosaccharides of DP $>6$ may form small helices that co-crystallise with starch and thereby increase retrogradation. Maltose was found to reduce retrogradation better than glucose, which can be of practical importance for bakery products.

\section{References}

Atkin, N. J., Abeysekera, R. M., \& Robards, A. W. (1998). The events leading to the formation of ghost remnants from the starch granule surface and the contribution of the granule surface to the gelatinization endotherm. Carbohydrate Polymers, 36, 193-204.

Bizot, H., Buléon, A., Mouhous-Riou, N., \& Multon, J. L. (1985). Some facts concerning water vapour sorption hysteresis on potato starch. In D. Simatos, \& J. L. Multon (Eds.), Properties of water in foods (pp. 83-93). Dordrecht: Martinus Nijhoff Publishers.

Bizot, H., Le Bail, P., Leroux, B., Davy, J., Roger, P., \& Buléon, A. (1997). Calorimetric evaluation of the glass transition in hydrated, linear and branched polyanhydroglucose compounds. Carbohydrate Polymers, 32, 33-50.

Chiotelli, E., Rolée, A., \& Le Meste, M. (2000). Effect of sucrose on the thermomechanical behavior of concentrated wheat and waxy corn starch-water preparations. Journal of Agricultural Food Chemistry, 48, 1327-1339.

Durán, E., Léon, A., Barber, B., \& Benedito de Barber, C. (2001). Effect of low molecular weight dextrins on gelatinization and retrogradation of starch. European Food Research and Technology, 212, 203-207.

Fredriksson, H., Silverio, J., Andersson, R., Eliasson, A. C., \& Åman, P. (1998). The influence of amylose and amylopectin characteristics on gelatinization and retrogradation properties of different starches. Carbohydrate Polymers, 35, 119-134.

Gérard, C., Planchot, V., Colonna, P., \& Bertoft, E. (2000). Relationship between branching density and crystalline structure of A- and B-type maize mutant starches. Carbohydrate Research, 326, 130-144.

Gidley, M. J., \& Bulpin, P. V. (1987). Crystallisation of maltooligosaccharides as models of the crystalline forms of starch: Minimum chain-length requirement for the formation of double helices. Carbohydrate Research, 161, 291-300.

Gudmundsson, M. (1994). Retrogradation of starch and the role of its components. Thermochimica Acta, 246, 329-341.

Hizukuri, S. (1985). Relationship between the distribution of the chain length of amylopectin and the crystalline structure of starch granules. Carbohydrate Research, 141, 295-306.

Imberty, A., Buléon, A., Tran, V., \& Pérez, S. (1991). Recent advances in knowledge of starch structure. Starch/Stärke, 43, 375-384.

Ispas-Szabo, P., Ravenelle, F., Hassan, I., Preda, M., \& Mateescu, M. A. (2000). Structure-properties relationship in cross-linked high-amylose starch for use in controlled release. Carbohydrate Research, 323, $163-175$.

Kalichevsky, M. T., Jaroszkiewicz, E. M., \& Blanshard, J. M. V. (1993). A study of the glass transition of amylopectin-sugar mixtures. Polymer, 34, 346-358.

Kalichevsky, M. T., Orford, P. D., \& Ring, S. G. (1990). The retrogradation and gelation of amylopectins from various botanical sources. Carbohydrate Research, 198, 49-55.

Katsuta, K., Nishimura, A., \& Miura, M. (1992a). Effects of saccharides on stabilities of rice starch gels: 1. Mono-and disaccharides. Food Hydrocolloids, 6, 387-398.

Katsuta, K., Nishimura, A., \& Miura, M. (1992b). Effects of saccharides on stabilities of rice starch gels: 2. Oligosaccharides. Food Hydrocolloids, 6, 399-408

Kohyama, K., \& Nishinari, K. (1991). Effect of soluble sugars on gelatinization and retrogradation of sweet potato starch. Journal of Agricultural Food Chemistry, 39, 1406-1410.

Krog, N., Oleson, S. K., Toernaes, H., \& Joensson, T. (1989). Retrogradation of the starch fraction in wheat bread. Cereal Foods World, 34 , $281-285$.

Léon, A., Durán, E., \& Benedito de Barber, C. (1997). Firming of starch gels and amylopectin retrogradation as related to dextrin production by $\alpha$-amylase. Zum Lebensmittel Untersuch und Forschung A, 205, $131-134$.

Levine, H., \& Slade, L. (1991). Water relationships in food. New York: Plenum Press.

Lourdin, D., Coignard, L., Bizot, H., \& Colonna, P. (1997). Influence of equilibrium relative humidity and plasticizer concentration on the water content and glass transition of starch materials. Polymer, 38, $5401-5406$

Miyajima, M., Koshika, A., Okada, J., Ikeda, M., \& Nishimura, K. (1997). Effect of polymer crystallinity on papaverine release from poly(L-lactic acid) matrix. Journal of Controlled Release, 49, 207-215.

Nilsson, G. S., Richardson, S., Huber, A., Torto, N., Laurell, T., \& Gorton, L. (2001). Microdialysis clean-up and sampling in enzyme-based methods for the characterisation of starch. Carbohydrate Polymers, 46, 59-68.

Nirkko, P., Gál, S., Giovanoli, R., \& Signer, R. (1975). Der einfluß des polymerisationsgrades von oligosacchariden auf die wasserdampfsorption und ihre hysterese. Starch/Stärke, 27, 278-280.

Silverio, J., Fredriksson, H., Andersson, R., Eliasson, A. C., \& Åman, P. (2000). The effect of temperature cycling on the amylopectin retrogradation of starches with different amylopectin unit-chain length distribution. Carbohydrate Polymers, 42, 175-184.

Sugiyama, H., Nitta, T., Horii, M., Motohashi, K., Sakai, J., Usui, T., 
Hisamichi, K., \& Ishiyama, J. (2000). The conformation of $\alpha$-(1 $\rightarrow 4)$-linked glucose oligomers from maltose to maltoheptaose and short-chain amylose in solution. Carbohydrate Research, 325, 177-182.

van Soest, J. J. G., de Wit, D., Tournois, H., \& Vliegenthart, J. F. G. (1994) The influence of glycerol on structural changes in waxy maize starch as studied by FT-IR spectroscopy. Polymer, 35, 4722-4727.

van Soest, J. J. G., Tournois, H., de Wit, D., \& Vliegenthart, J. F. G. (1995).
Retrogradation of potato starch as studied by Fourier transform infrared spectroscopy. Starch/Stärke, 46, 453-457.

Waigh, T. A., Donald, A. M., Heidelbach, F., Riekel, C., \& Gidley, M. J. (1999). Analysis of the native structure of starch granules with small angle X-ray microfocus scattering. Biopolymers, 49, 91-105.

Yadav, S. K., Khilar, K. C., \& Suresh, A. K. (1997). Release rates from semi-crystalline polymer microcapsules formed by interfacial polycondensation. Journal of Membrane Science, 125, 213-218. 